\title{
Automatic Particle Coordination Recognition Using Principal Component Analysis and Kohonen Neural Nets
}

\author{
J.P. Simmons*, D.M. Dimiduk*, M. DeGraef** \\ *Materials and Manufacturing Directorate, AFRL/MLLMD, Wright-Patterson AFB, OH 45433 \\ **Department of MS\&E, Carnegie Mellon University, Pittsburgh, PA 15213
}

Materials science is rapidly becoming a computationally intensive field, fueled not only by the traditional modeling and simulation efforts, but also from the ability to automate techniques for data acquisition and distribution. In particular, with the emerging rapid data generation techniques such as Orientation Imaging Microscopy (OIM) or Focused Ion Beam (FIB), large rich datasets can be acquired rapidly, providing data fidelity never before realized. This creates the condition in which data is collected much more rapidly and will outstrip the ability of traditional analysis methods. Towards this end, we are developing methods to automatically inspect and analyze datasets. In this work, we develop a technique that automatically classifies particles in a sampled microstructure, according to their coordination with other particles. Particles are extracted from 2-D images, using cross-correlation[1]. An unbiased representation of the particle images is developed with Principal Component Analysis (PCA)[2]. From this, a Kohonen Neural Net[3], also referred to as a Self Organizing Map (SOM), is used to classify particles into groups with similar coordination. Although the technique is applicable to experimental images, simulated images of a Ni-base superalloy are used, in order to minimize image correction difficulties.

Images were simulated with a 2-D Phase Field model that was calibrated to mimic Ni-base superalloys.[4] A "typical particle" was chosen as a kernel for cross-correlation to locate other particles in this size class. Small 41x41 pixel images were cut, centered about each of these particles. Typical particle images are given in Figure 1. In all, 282 particle images were collected.

Modeling pixel intensities as random numbers, the covariances of individual pixel pairs over the stack of particle images were computed. These correlations were decoupled into principal components of correlation using PCA. The first 10 principal components of correlation are given in Figure 2. This provided a sample-dependent representation of the particle images. It was found that an expansion using the first 5 principal components of coordinations reproduced all of the important features of particle coordination; This is shown in Figure 3.

Thus, all particle images couild be represented in a 5-dimensional space. The particle representations in this space were then classified using a SOM. With the SOM, similar representations of the particle images cluster together in a 2-dimensional map, a section of which is showin in Figure 4. The SOM was then divided into regions of similar behavior, schematically depicted in Figure 5. Within each of the regions in this schematic, particles of similar coordination were clustered. Examples of this clustering are given in Figure 6, which shows selected particles from the upper left and center left regions in the SOM. All single particles collected in the upper left and all particles with neighboring particles immediately below clustered in the center left region.

With this technique, it is possible to automatically analyze a microstructure in terms of particle coordination types. Typical cases can be identified by large numbers of particles collecting into specific regions and rare events by regions containing only one or two particles.

[1] R.C. Gonzalez and R.E. Woods, Digital Image Processing, Addison-Wesley, NY, 1992.

[2] I.T. Jolliffe, Principal Component Analysis, 2nd ed., Springer, NY, 2002.

[3] T. Kohonen, Self-Organizing Maps, 3rd ed., Springer, NY, 1995.

[4] Y. Wen, et al., Acta Mater. 51 (2003) 1123. 


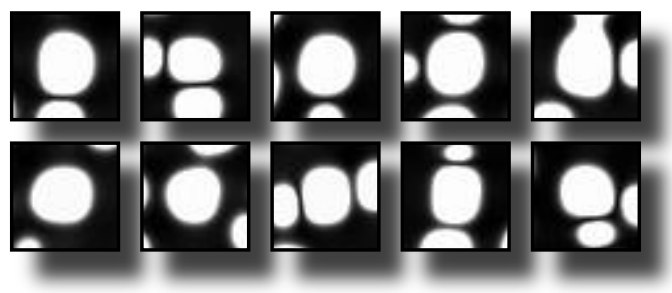

Figure 1. Typical particles extracted from simulated Ni-base alloy mictostructure.

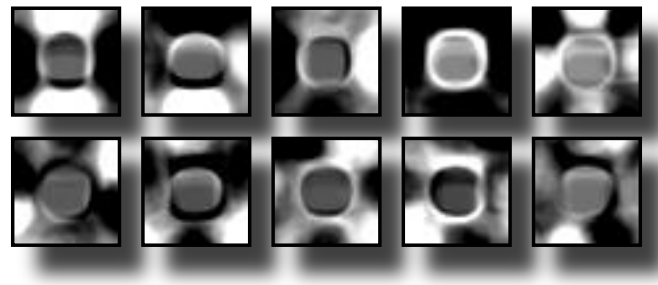

Figure 2. First 10 principal components of correlation, consisting of collections of pixels that tend to vary as highly correlated groups.

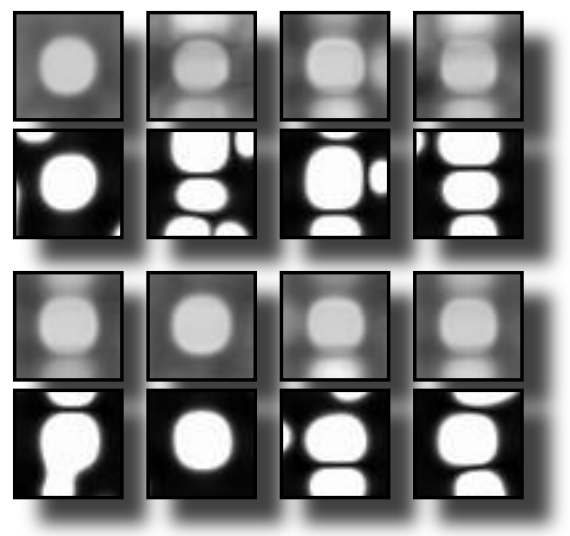

Figure 3. 5 term reconstructions of images (top and third rows), along with the original images (second and fourth rows).

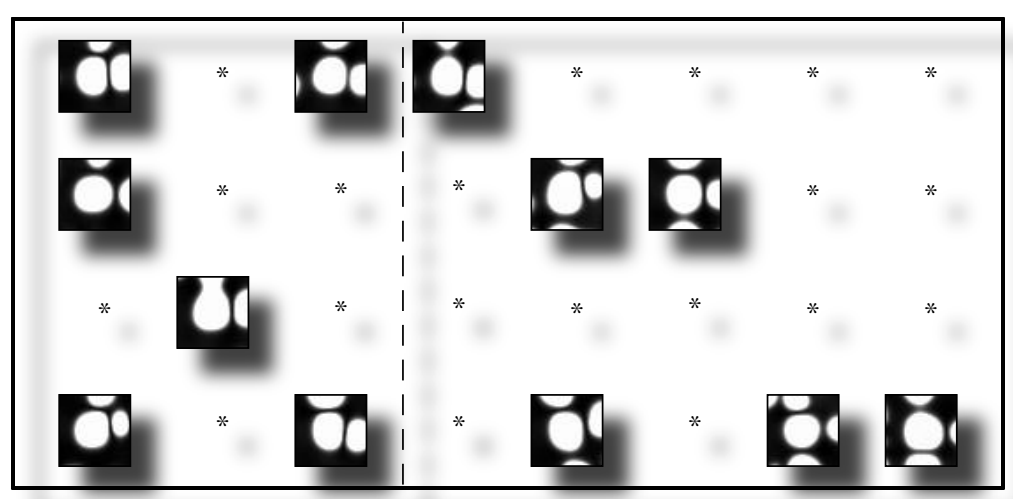

Figure 4. Section of a SOM, showing regions of clustering of particles with similar coordinations.

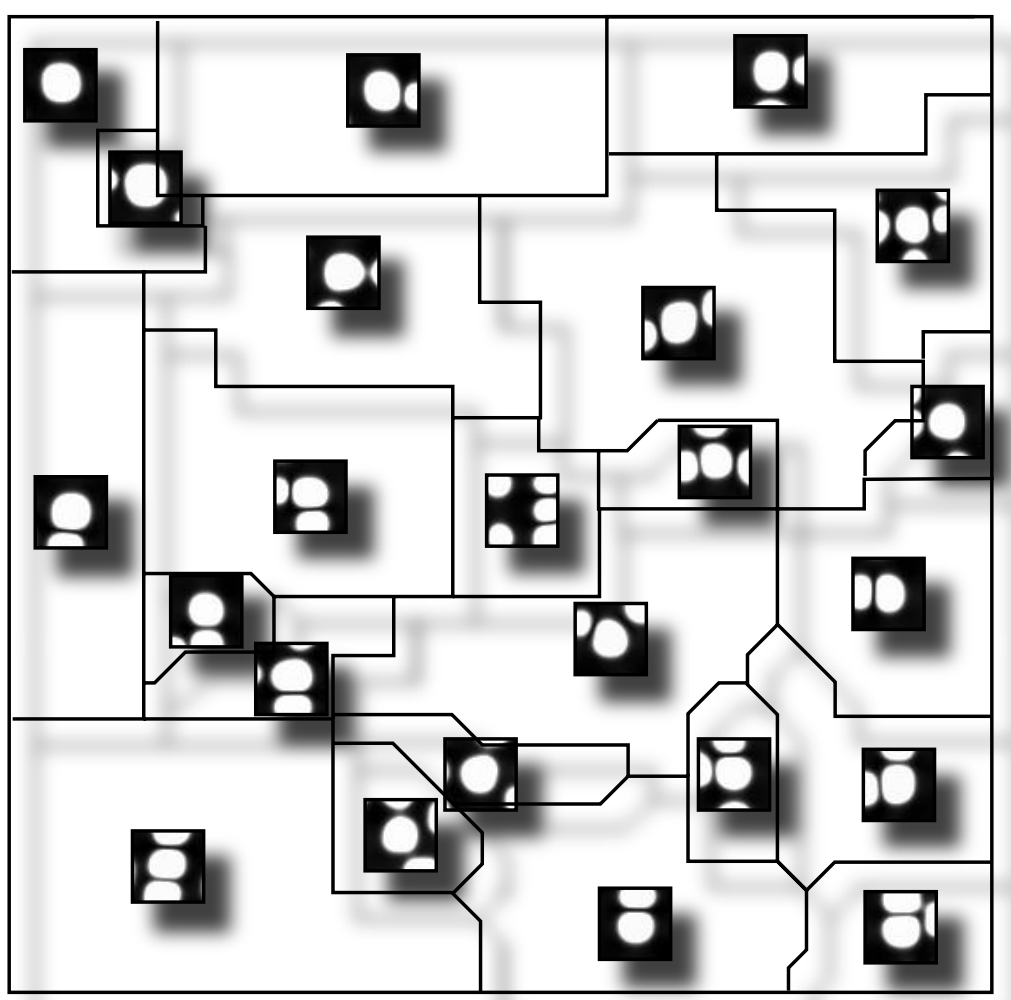

Figure 5. Schematic illustration of the SOM obtained in this study, showing regions of distinct particle coordinations.

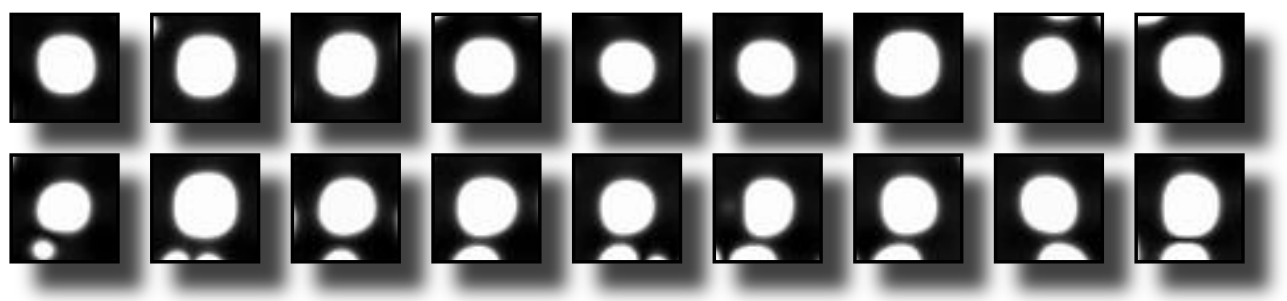

Figure 6. Particles from regions of the SOM schematic shown in Figure 5. Particles from the upper left region in Figure 5 (top row). Particles from the center left region of Figure 5 (bottom row). 